\title{
Mass-producible micro-optical elements by injection compression molding and focused ion beam structured titanium molding tools
}

\author{
Simon Ristok, ${ }^{1,}{ }^{*}$ Marcel Roeder, ${ }^{2}$ Simon Thiele, ${ }^{3}$ Mario Hentschel, ${ }^{1}$ \\ Thomas Guenther, ${ }^{2,4}$ André Zimmermann, ${ }^{2,4}$ Alois M. Herkommer, ${ }^{3}$ AND \\ HARALD GIESSEN ${ }^{1}$ \\ ${ }_{1}^{1} 4^{\text {th }}$ Physics Institute and Research Center SCOPE, University of Stuttgart, Pfaffenwaldring 57, 70569 Stuttgart, Germany \\ ${ }^{2}$ Hahn-Schickard, Allmandring 9b, 70569 Stuttgart, Germany \\ 3 Institute for Applied Optics (ITO) and Research Center SCOPE, University of Stuttgart, Pfaffenwaldring 9, 70569 Stuttgart, Germany \\ ${ }^{4}$ Institute for Micro Integration (IFM), University of Stuttgart, Allmandring 9b, 70569 Stuttgart, Germany \\ *Corresponding author: s.ristok@pi4.uni-stuttgart.de
}

\begin{abstract}
We demonstrate mass production compatible fabrication of polymer-based micro Fresnel lenses by injection compression molding. The extremely robust titanium molding tool is structured with high precision by focused ion beam milling. In order to achieve optimal shape accuracy in the titanium we use an iterative design optimization. The inverse Fresnel lens structured into the titanium is transferred to polymers by injection compression molding, enabling rapid mass replication. We show that the optical performance of the molded diffractive Fresnel lenses is in good agreement with simulations, rendering our approach suitable for applications which require compact and high quality optical elements in large numbers. (C) 2020 Optical Society of America
\end{abstract}

http://dx.doi.org/10.1364/OL.385599

Injection molded polymers are the material of choice for microoptics used in mass-producible devices such as smartphones or optical sensors [1-3]. Hundreds of millions of such micro-optical elements are manufactured every year, using a so-called LIGA process (German "Lithographie, Galvanoformung, Abformung") where a silicon (Si) master is fabricated by grey-scale electron beam lithography and subsequent etching. An electroplating process is then used to transfer the structured $\mathrm{Si}$ surface into a solid nickel (Ni) shim, which serves as mold in the following replication step. Techniques like injection (compression) molding [4,5], hot embossing [6] or nanoimprint lithography $[7,8]$ are commonly used for the replication in polymers $[9,10]$. In total, two inversion steps are necessary to transfer the structure from the Si master to a plastic polymer part. For injection molding, a polymer is heated, injected into the Ni shim, pressurized, and released. A Ni shim usually lasts for about 10,000 repetitions before it has to be replaced. An alternative method to fabricate micro-optical elements is nanoimprint lithography. However, this method also requires two inversion steps as well as photopolymers that react to UV light. This can easily leave a yellowish hue in the micro-optics due to residual photoinitiator [11] and might render the plastic devices prone to degradation and aging.
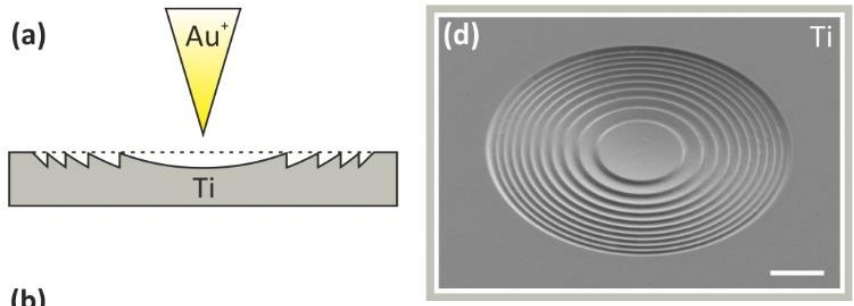

(b)
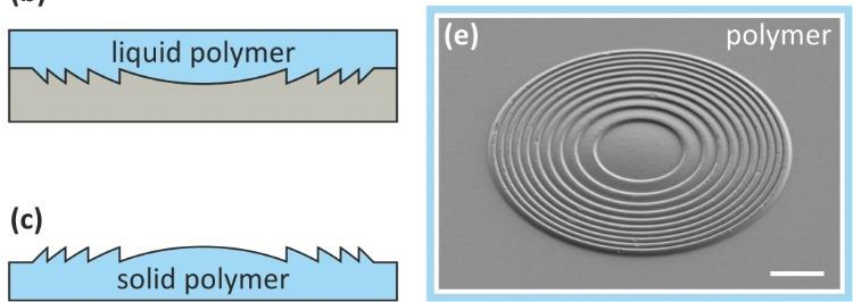

Fig. 1. Workflow diagram. (a) FIB structuring of inverse geometry in Ti. (b) Injection compression molding in transparent polymer. (c) Solid polymer Fresnel lens. (d),(e) SEM images of Ti mold insert and polymer Fresnel lens. Scalebars: $20 \mu \mathrm{m}$. 
Here, we introduce an alternative method that requires only a single inversion step and works with clear plastics without photoinitiator, avoiding a UV curing step. A non-magnetic titanium master (Ti-6Al-4V Grade 5) is structured directly using focused ion beam (FIB) milling [12] and is subsequently used as mold insert for injection compression molding. While the surface quality should be similar to structures fabricated with the commonly used electroplated Ni shims, Titanium (Ti) as extremely hard material should minimize the wear from the molding step, resulting in a large number of replications before it needs to be replaced. We make use of the fact that FIB milling is an intrinsic "grey-scale process" with potentially thousands of levels, as the material etch rate depends directly on the ion dose (in contrast to UV and electron beam greyscale lithography). We demonstrate the manufacturing and characterization of the Ti mold insert and show the imaging performance of diffractive Fresnel lenses made by injection compression molding. As Fresnel lenses are often used in various micro-optical applications [13-15], they are well suited to point out the benefits and the limitations of the fabrication process. The lenses have a diameter of $100 \mu \mathrm{m}$, a focal length of $200 \mu \mathrm{m}$, and are designed for a target wavelength of $550 \mathrm{~nm}$.

The fabrication process is outlined in Fig. 1. First, the inverse profile of the Fresnel lens is milled by a focused beam of singly charged Gold $\left(\mathrm{Au}^{+}\right)$ions (Fig. 1(a)) into the Ti mold insert polished to optical quality. To avoid drifting of the mold insert due to thermal expansion, it is transferred into the vacuum chamber of the FIB machine (Raith ionLine Plus) $24 \mathrm{~h}$ prior to the actual structuring process. The ion dose is not deposited in a single step, but in 130 successive structuring cycles to avoid nonlinear milling effects. During each cycle the ion beam is scanned in concentric circles of increasing diameter over the $\mathrm{Ti}$ surface in order to prevent directionally dependent deviations. As the diameter of the structure is $100 \mu \mathrm{m}$ and its maximum depth is around $1 \mu \mathrm{m}$, the volume which has to be removed by FIB milling is comparably large, resulting in a structuring duration of $\sim 24 \mathrm{~h}$. The comparably long structuring times are also related to the high resolution and form accuracy afforded by our method. Larger diameters and deeper features are possible, if longer fabrication times can be accepted.
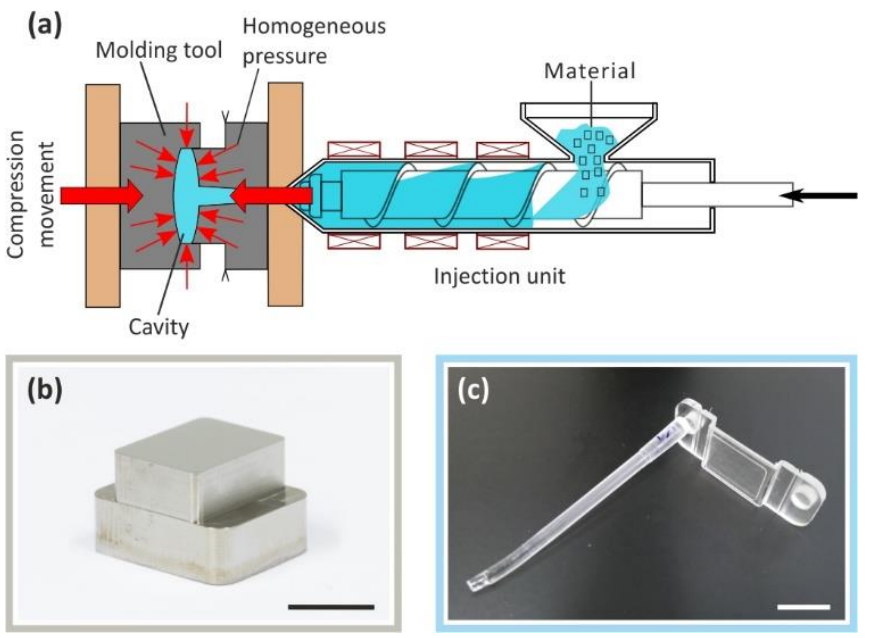

Fig. 2. Injection compression molding. (a) Principle. (b) Ti molding tool. (c) Polymer part after molding. Scalebars: $1 \mathrm{~cm}$.
In the next step the structures milled into the Ti surface are replicated in polymers by injection compression molding (Fig. 1(b)). The Ti mold insert is integrated into an injection compression molding tool, into which the plasticized polymer Zeonex 330R is injected. After the polymer has cooled and solidified the molded sample can be removed and characterized (Fig. 1(c)). Scanning electron microscope (SEM) images of the structured Ti surface and the molded polymer Fresnel lens are shown in Fig. 1(d, e).

The injection molding process is illustrated in more detail in Fig. 2(a). The transparent polymer is heated to $255^{\circ} \mathrm{C}$ and injected into the molding cavity, which is preheated to $120^{\circ} \mathrm{C}$. The injection pressure is 1500 bar. Unlike in classical injection molding, the molding tool is initially not closed completely during the injection phase. With a defined delay after the material injection, a moveable compression stamper in the molding tool is used to shape the molded part into its final dimensions. Pressure applied this way is very homogeneous throughout the entire cavity. Therefore, less residual stress remains within the solid plastic part, resulting in reduced birefringence compared to parts made by standard injection molding [16]. Less birefringence, in turn, usually leads to superior imaging properties. The time needed for one replication is $26 \mathrm{~s}$. A photograph of the used Ti mold insert is shown in Fig. 2(b). The inverse Fresnel lenses are milled into the polished surface on top. After the molding process the sample is prepared for further characterization by removing the excess waste plastic parts visible in Fig. 2(c). The resulting part has a thickness of $900 \mu \mathrm{m}$ and measures $1.3 \times 1.3 \mathrm{~cm}^{2}$. No further post process treatment is required, and the sample can directly be examined by optical microscopy and atomic force microscopy (AFM) to verify the quality of the replication process.

An essential prerequisite for the shape accuracy of the injection molded structures is the shape accuracy of the inverse structures
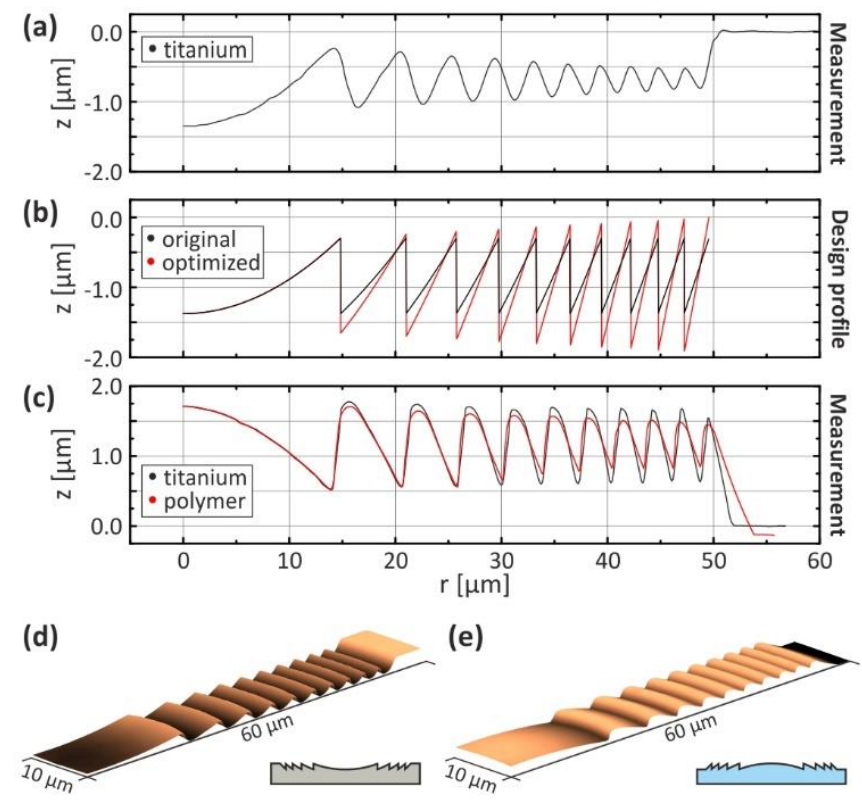

Fig. 3. Shape accuracy measurements. (a) Profile of inverse Fresnel lens on Ti mold insert. (b) Original and optimized design profile. (c) Comparison of optimized profile in Ti and polymer. (d),(e) AFM measurements of Ti mold insert and polymer Fresnel lens. 
milled into the Ti molding tool. Therefore, we examine the Ti mold insert after FIB milling with a confocal microscopy technique (Nanofocus $\mu$ surf expert) to measure the profile of the inverse Fresnel lens and characterize and improve the fabrication results.

As a first step, we mill the target design, assuming a one-to-one correspondence between deposited dose and milled structure depth, into the Ti mold insert. The profile in Fig. 3(a) indicates that the depth of the central part is $1.11 \mu \mathrm{m}$, which is close to the design depth of $1.18 \mu \mathrm{m}$. However, the height difference between adjacent peaks and valleys is significantly reduced towards the outer boundary of the lens. Possible reasons are the finite beam size and defocusing due to the significant depth of the structure [17]. The steps are most critical features in the diffractive Fresnel lens design as regions with maximum and zero ion doses are in direct vicinity of each other. If the size of the ion beam is too large, part of the high dose might impinge on the region where no dose is supposed to go, reducing the step height and rounding the sharp edge of the step. Furthermore, the ion beam is initially focused at the top of the Ti surface and is not refocused when milling into deeper lying regions. This could lead to defocusing with increasing depth, resulting in a reduced milling rate and therefore causing deviations from the designed depth and shape. It is, however, challenging to determine how these different effects impact the milling process quantitatively. For this reason, we refrain from tuning the process parameters (e.g., focusing or beam size). Instead we incorporate the measured deviations into the milling design to iteratively approach the target design in the Ti mold insert. The original and optimized designs are displayed in Fig. 3(b). In the original design the step height is constant over the entire profile. Deviations visible in Fig. 3(a) are evaluated and used to generate the optimized structuring design. The radial positions and height values of the minima and maxima are extracted. We use linear fits to calculate the slopes which describe the behavior of the minima and maxima separately. For the maxima, the first peak at the border of the central spherical region serves as reference point, where the deviation is set to zero. For the minima, the depth in the center is used as reference point. The deviations at the step positions are calculated and added to the original design, resulting in an increase of the step height towards the outer parts of the structure.

The optimized design is FIB milled into the Ti molding tool and the profile is measured with atomic force microscopy (Veeco Dimension Icon, AFM probe: Tap300Al-G (BudgetSensors)) (Fig. $3(\mathrm{c})$ ). The profile is mirrored horizontally to facilitate comparison with the profile of the molded polymer lens, plotted in black. Compared to Fig. 3(a) the decrease of the step height is strongly reduced. Generally, the milled structure is very close to the ideal Fresnel lens design. The rounded edges are, however, still visible. Another optimization step where the shape of each segment of the Fresnel lens is measured and optimized separately could help to minimize this effect.

As a next step, we compare the quality and shape accuracy of the polymer replication process. The profile of the replicated polymer lens, again determined by AFM measurement, is shown in red in Fig. 3 (c). In the central part the agreement between mold insert and molded lens is good, whereas some smaller deviations can be seen for the finer structures in the outer parts. We attribute this mainly to the shrinkage of the polymer during cool-down. Both profiles are taken from the AFM measurements shown in Fig. 3(d, e).

While high shape accuracy is a suitable indicator for the quality of the molded Fresnel lenses, their optical performance is ultimately the most important benchmark. An overview of the optical setup used to characterize the imaging properties is shown in Fig. 4(a). A LED white light source is used for illumination. The light is collimated and then sent through a $550 \mathrm{~nm}$ filter $(\Delta \lambda=40 \mathrm{~nm})$, as this is the wavelength the diffractive Fresnel lens is designed for. Homogeneous radiant intensity is achieved by using a diffuser plate. An inverse USAF 1951 resolution test target is used as test object.
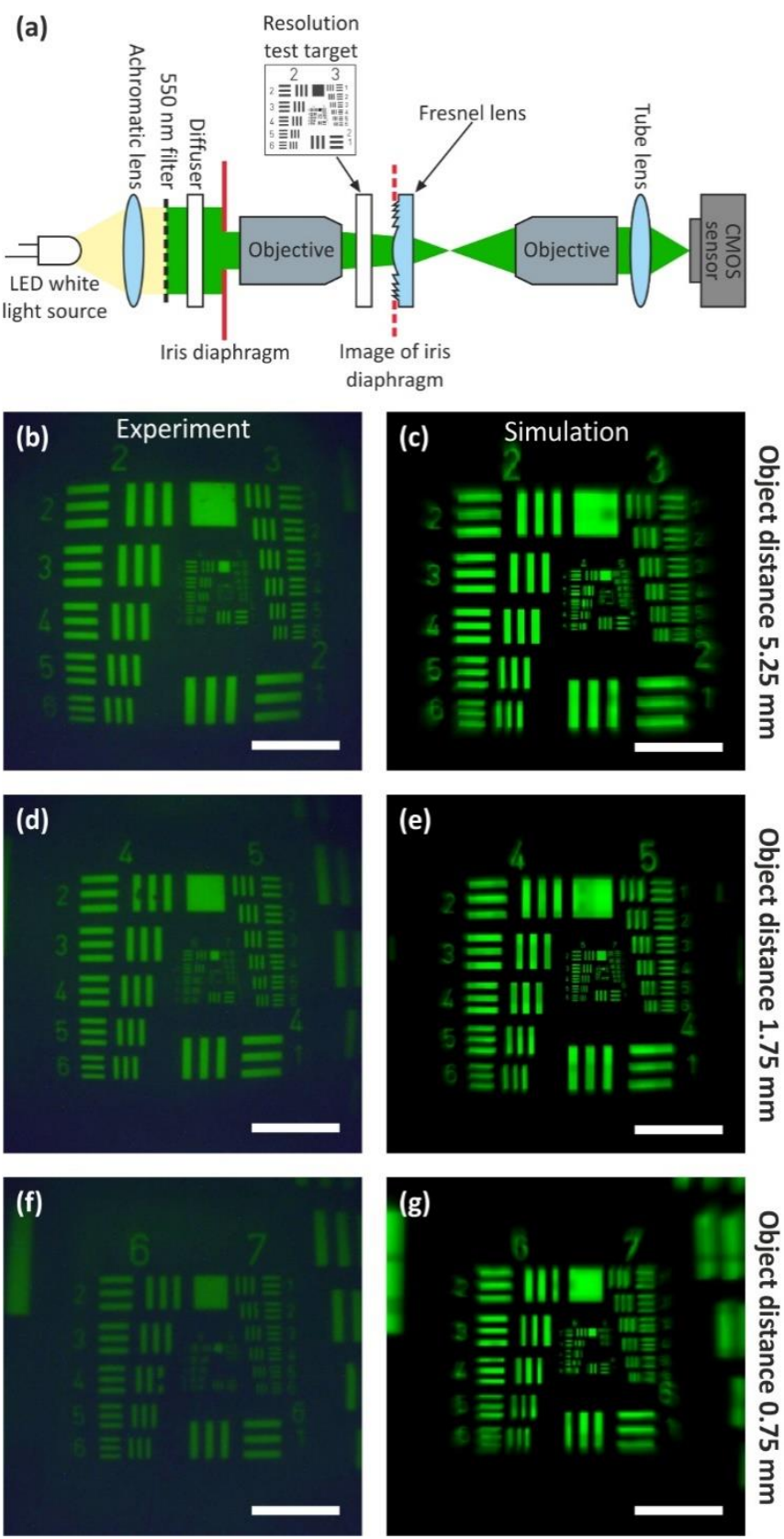

Fig. 4. Imaging performance. (a) Optical setup used for resolution test measurements. (b)-(g) Measured and simulated images of USAF 1951 test target for distances 5.25, 1.75 and $0.75 \mathrm{~mm}$ from the Fresnel lens. (b),(d) and (f) are measurements, (c),(e) and (g) corresponding simulations. Scalebars: $50 \mu \mathrm{m}$. 
The light is focused onto the Fresnel lens by a microscope objective and the resolution test target is placed between objective and Fresnel lens. The imaging plane of the Fresnel lens is viewed by a standard microscopy setup (objective, tube lens and CMOS sensor). To minimize unwanted stray light, an adjustable iris diaphragm is added to the illumination and placed between light source and objective. The exact position is chosen such that an image of the iris is projected onto the Fresnel lens. The size of the iris is then adjusted to match the diameter of the lens, which has the same effect as a physical aperture. In Fig. 4(b)-(g) the experimental imaging quality is compared to simulations. Fig. 4(b, d, f) show the resolution test target imaged by the replicated Fresnel lens using monochromatic illumination. The distance between target and Fresnel lens is varied ( $5.25 \mathrm{~mm}, 1.75 \mathrm{~mm}, 0.75 \mathrm{~mm}$ ) to determine the smallest visible features. To facilitate evaluation by the reader the contrast of the images was subsequently enhanced. In addition, we simulate the imaging performance of the ideal diffractive Fresnel lens design for the different object distances using the built-in 'Image Simulation' tool of the raytracing software ZEMAX 13 (Fig. 4 (c, e, g)). In Fig. 4(b) the image of groups 2 and 3 of the test target is shown. A barrel distortion is present both in the measurementand in the simulation. Fig. 4(d) shows groups 4 and 5, with the smaller groups 6 and 7 in the center. There is good agreement between experiment and simulation for the imaging of groups 4 and 5. While all elements of group 6 can be distinguished in the simulation, this is only possible up to element 2 in the measurement. When the target is placed very close $(0.75 \mathrm{~mm})$ to the lens, groups 6 and 7 can be distinguished, which indicates a resolution down to $2.5 \mu \mathrm{m}$, which can be expected from our 0.24 NA Fresnel lens design at $550 \mathrm{~nm}$. The imaging quality is similar for experiment and simulation, but there is some slight mismatch in magnification.

In conclusion, we have presented an alternative approach for the fabrication of a master for mass replication of micro-optical elements. The Ti master was structured directly by FIB milling, avoiding the necessity of a second inversion step. We used the measured profile of the milled Fresnel lens to iteratively optimize the structuring process. After optimization, the Ti master was used as mold insert for injection compression molding. AFM measurements confirmed the good agreement of the inverse Fresnel lens in the molding tool and the replicated polymer lens, with some minor deviations. Those deviations could most probably be reduced by optimizing the injection compression molding process, e.g., by using a hot runner system and a variothermal [5] mold temperature control. The imaging performance of the diffractive Fresnel lenses was demonstrated using a standard resolution test target. In contrast to the standard mold fabrication processes, such as diamond turning, FIB milling offers a multitude of degrees of freedom in the optical design. It is thus very intriguing to use the described fabrication process for the realization of advanced optical designs, e.g., aspheric Fresnel lenses or nonrotationally symmetric free-form geometries. We envision the use of this technique also for more complex polymer optics in combination of refractive and diffractive components [18,19].
Funding. Bundesministerium für Bildung und Forschung (13N14097, PRINTOPTICS, PRINTFUNCTION), ERC Advanced Grant (COMPLEXPLAS, 3DPRINTEDOPTICS), Baden-Württemberg Stiftung (OPTERIAL).

Disclosures. The authors declare that there are no conflicts of interest related to this article.

\section{References}

1. M. Sortino, G. Totis, and E. Kuljanic, "Comparison of injection molding technologies for the production of micro-optical devices," Procedia Eng. 69, 1296-1305 (2014).

2. H. N. Hansen, R. J. Hocken, and G. Tosello, "Replication of micro and nano surface geometries," CIRP Ann. - Manuf. Technol. 60, 695-714 (2011).

3. M. Roeder, T. Guenther, and A. Zimmermann, "Review on fabrication technologies for optical mold inserts," Micromachines 10, 233 (2019).

4. M. Roeder, M. Drexler, T. Rothermel, T. Meissner, T. Guenther, and A. Zimmermann, "Injection compression molded microlens arrays for hyperspectral imaging," Micromachines 9, 355 (2018).

5. C. Rytka, P. M. Kristiansen, and A. Neyer, "Iso- and variothermal injection compression moulding of polymer micro- and nanostructures for optical and medical applications," J. Micromechanics Microengineering 25, (2015).

6. T. Mappes, M. Worgull, M. Heckele, and J. Mohr, "Submicron polymer structures with X-ray lithography and hot embossing," Microsyst. Technol. 14, 1721-1725 (2008).

7. Y. Yao, H. Liu, Y. Wang, Y. Li, B. Song, A. Bratkovsk, S. Y. Wang, and W. Wu, "Nanoimprint lithography: an enabling technology for nanophotonics," Appl. Phys. A Mater. Sci. Process. 121, 327-333 (2015).

8. Y. Kanamori, M. Okochi, and K. Hane, "Fabrication of antireflection subwavelength gratings at the tips of optical fibers using UV nanoimprint lithography," Opt. Express 21, 322 (2013).

9. S. Kang, Micro/Nano Replication: Processes and Applications (John Wiley \& Sons, Incorporated, 2012).

10. M. T. Gale, "Replication techniques for diffractive optical elements," Microelectron. Eng. 34, 321-339 (1997).

11. T. Gissibl, S. Thiele, A. Herkommer, and H. Giessen, "Two-photon direct laser writing of ultracompact multi-lens objectives," Nat. Photonics 10, 554-560 (2016).

12. F. Watt, A. A. Bettiol, J. A. van Kan, E. J. Teo, and M. B. H. Breese, "lon beam lithography and nanofabrication: a review," Int. J. Nanosci. 4, 269 286 (2005).

13. J. E. Hergert, D. J. Glugla, A. C. Sullivan, M. D. Alim, and R. R. McLeod, "High efficiency Fresnel lens design and fabrication in a two-stage photopolymer," Opt. Lett. 44, 1540 (2019).

14. S. Thiele, C. Pruss, A. M. Herkommer, and H. Giessen, "3D printed stacked diffractive microlenses," Opt. Express 27, 35621-35630 (2019).

15. D. Loaldi, D. Quagliotti, M. Calaon, P. Parenti, M. Annoni, and G. Tosello, "Manufacturing signatures of injection molding and injection compression molding for micro-structured polymer fresnel lens production," Micromachines 9, 653 (2018).

16. S. Y. Yang and M. Z. Ke, "Experimental study on the effects of adding compression to injection molding process," Adv. Polym. Technol. 14, 1524 (1995).

17. P. Scholz, U. Kerst, C. Boit, T. Kujawa, and T. Lundquist, "Instant solid immersion lens creation in silicon with a focused ion beam - Comparing refractive and diffractive methods," Conf. Proc. from Int. Symp. Test. Fail. Anal. 46-53 (2011).

18. H. P. Herzig, ed., Micro-Optics: Elements, Systems and Applications (Taylor \& Francis, 1997).

19. H. Zappe, Fundamentals of Micro-Optics (Cambridge University Press, 2010). 